\title{
Perfil socioeconômico, nutricional e de saúde de adolescentes recém-ingressos em uma universidade pública brasileira
}

\section{Socioeconomic, nutritional and health profile of adolescents recently admitted to a Brazilian public university}

Valéria Cristina Ribeiro VIEIRA ${ }^{1}$

Sílvia Eloiza PRIORE²

Sônia Machado Rocha RIBEIRO²

Sylvia do Carmo Castro FRANCESCHINI ${ }^{2}$

Laerte Pereira ALMEIDA²

\section{RE S U M O}

Objetivou-se delinear o perfil socioeconômico, nutricional e de saúde de adolescentes recém-ingressos em uma universidade pública brasileira. As variáveis comportamentais foram obtidas por meio de questionário, 0 percentual de gordura corporal (\% GC) pelo somatório das dobras cutâneas e o estado nutricional pelo Índice de Massa Corporal. A maioria dos adolescentes era do sexo feminino (57,3\%), não residia com familiares $(89,8 \%)$, consumia bebida alcoólica $(73,5 \%)$, omitia alguma refeição principal $(57,3 \%)$ e rejeitava um ou mais alimentos do grupo das hortaliças (79,5\%). Cerca de 57,0\% não realizavam atividade física e 7,0\% fumavam. Em torno de $72,0 \%$ e $25,0 \%$, respectivamente, consumiam hortaliça e fruta cinco ou mais vezes na semana. Os eutróficos predominavam, mas $58,7 \%$ destes apresentavam \% GC elevado. Concluiu-se que considerável parcela dos indivíduos estudados residia sem os familiares e apresentava, além de inadequação da composição corporal e do comportamento alimentar, outros fatores de risco à saúde, como o consumo de álcool e a inatividade física.

Termos de indexação: adolescência, comportamento alimentar, índice de massa corporal, composição corporal, antropometria.

\section{A B S T R A C T}

The objective of this work was to describe the socioeconomic, nutritional and health profile of adolescents recently admitted to a Brazilian public university. The behavioral variables were obtained by interview, the body

\footnotetext{
1 Bolsista PIBIC/CN Pq, Curso de N utrição, U niversidade Federal de Viçosa.

2 Departamento de Nutrição e Saúde, Universidade Federal de Viçosa. Av. PH Rolfs, s/n, 36571-000, Viçosa, MG, Brasil. Correspondência para/Correspondence to: S.E. PRIORE.
} 
fat percentage (BF\%) by the sum of the four skinfold thicknesses and the nutritional status by body mass index. Most of the students were female $(57.3 \%)$, lived away from their families $(89.8 \%)$, drank alcohol $(73.5 \%)$, skipped a main meal $(57.3 \%)$ and rejected one or more types of vegetables $(79.5 \%)$. About $57.0 \%$ did not practice any physical activity and $7.0 \%$ smoked. About $72.0 \%$ and $25.0 \%$, respectively, consumed vegetable and fruit five times or over during the week. The eutrophic subjects predominated, but $58.7 \%$ of them presented a high BF\%. The results showed that a considerable number of the subjects lived away from their families and had inadequate body composition and feeding behavior, besides other risks to their health, such as alcohol drinking and physical inactivity.

Index terms: adolescence, feeding behavior, body mass index, body composition, anthropometry.

\section{N T R O D U Ç Ã O}

Segundo a Organización M undial... (1977), a adolescência abrange a faixa etária entre $10 \mathrm{e}$ 20 anos, e após os 17 anos é considerada por Souza (1989) como adolescência tardia. Nesta fase, geralmente, observam-se determinadas condições características do final desse período de vida, como estabelecimento da identidade sexual, entrosamento com os vínculos de trabalho, criação de sistema pessoal de valores morais, capacidade de manter relações amorosas e retorno ao relacionamento mais estável com os pais (Bastos, 1992).

Existem, contudo, muitas variações individuais - influenciadas por fatores genéticos ou socioculturais - as quais tornam os limites etários dos estágios da adolescência aproximados. A crescente complexidade do fenômeno adolescente tem ultrapassado os limites da segunda década, pois o desenvolvimento tecnológico da sociedade ocidental tem levado a um prolongamento deste processo transicional. Isso contribui para que os adolescentes na fase final ou tardia nem sempre tenham características adultas bem definidas (Chipkevitch, 1995).

A proximidade com a vida adulta pode proporcionar oportunidades finais para implementar atividades visando prevenir problemas de saúde (World Health..., 1995). A adolescência, segundo Jacobson (1998), é o momento privilegiado para se colocar em prática medidas preventivas, uma vez que os hábitos alimentares criados quando o indivíduo afirma sua independência, tornando-se responsável por suas próprias ingestões alimentares, aparentemente persistem na idade adulta.

A atenção a essa população reveste-se de maior relevância ao se ponderar sobre a afirmação de Knobel \& Santos (1991): “ se por um lado 0 adolescente adoece menos que outras faixas etárias, quando o faz, não raramente isso se dá de maneira sorrateira, insidiosa e dissimulada, levando-se em conta até a negação da doença por parte do próprio adolescente" . Neste sentido, paradigmas devem ser transferidos dos cuidados com a doença para a prevenção e a promoção da saúde, considerando também que as causas mais freqüentes de enfermidades entre adolescentes no mundo inteiro não são as infecções, mas os fatores sociais e comportamentais (Blum, 1997).

M odificações quanto ao padrão alimentar, prática de atividade física, stress, consumo de álcool e cigarros podem interferir na formação física e social, bem como nas condições nutricionais e na vulnerabilidade biológica desse grupo. De acordo com Jacobson (1998), além das ingestões alimentares inadequadas, outras situações freqüentes podem afetar 0 estado nutricional dos adolescentes, como: condições para aquisição, conservação e preparo dos alimentos; fatores psicossociais; doenças relacionadas à nutrição; e estilo de vida (abuso de álcool e fumo e um modo de vida sedentário).

Como os instrumentos de avaliação nutricional para esta faixa etária são ainda muito controversos, para amenizar os riscos de um exame 
inadequado, Frisancho (1989) recomenda que, junto aos critérios antropométricos, seja também analisada a composição corporal do indivíduo, de forma a se ter melhor precisão na avaliação nutricional, propiciando o desenvolvimento de atividades preventivas as quais envolvam da obesidade à anorexia e todas as suas conseqüências.

Somadas a situações próprias da adolescência, como intensas alterações biológicas e instabilidade psicossocial, mudanças oriundas do ingresso no meio universitário, como novas relações sociais e adoção de novos comportamentos, podem tornar os adolescentes universitários um grupo vulnerável a circunstâncias que colocam em risco sua saúde.

Neste contexto, um aspecto que pode interferir é o distanciamento da família, o qual comumente coincide com o ingresso na universidade. Esta situação pode ser encarada como um "grito de liberdade", pois é quando o adolescente tem a necessidade de assumir seus próprios atos, tornando-se responsável por si mesmo, e encontra indivíduos de todas as partes do país, com grande diversificação de hábitos, costumes e expectativas.

Os adolescentes constituíam cerca de $60 \%$ dos estudantes admitidos na universidade pública brasileira avaliada nos dois anos consecutivos em que este estudo foi desenvolvido. Assim, cresceu a necessidade de se conhecer as condições socioeconômicas, nutricionais e de saúde dessa população, pois os resultados da presente pesquisa podem subsidiar o estabelecimento de medidas de intervenção adequadas, visando melhorar a saúde atual e futura dos adolescentes universitários.

Desta forma, este estudo, objetivando traçar o perfil dos adolescentes de uma universidade pública brasileira, buscou conhecer as condições socioeconômicas e de saúde, a prática de atividade física, o consumo de álcool e cigarros e o comportamento alimentar do grupo; verificar as principais mudanças ocorridas após o ingresso na universidade, em relação à alimentação, atividade física, tabagismo e etilismo; e avaliar o estado nutricional e o percentual de gordura corporal dos estudantes.

\section{CASUÍSTICAE MÉTODOS}

A população de estudo constituiu-se de 185 adolescentes entre 18 e 19 anos, sendo 57,3\% do sexo feminino. Todos eram estudantes matriculados no primeiro ano de cursos de graduação de uma universidade pública brasileira. O período de estudo foi de setembro de 1998 a julho de 1999.

Todos os dados foram coletados apenas uma vez. As condições socioeconômicas, demográficas e de saúde e variáveis comportamentais foram coletadas por meio de questionário padronizado e previamente testado.

Em relação à antropometria, o peso foi obtido por meio de balança eletrônica, com capacidade de $150 \mathrm{~kg}$ e divisão de 50 gramas, segundo técnicas preconizadas por Jelliffe (1966); a estatura foi verificada com fita métrica metálica (M icrotoise - Stanley-M abo Ltd; CM S W eighting Equipment Ltd), com extensão de 2 metros, dividida em centímetros e subdividida em milímetros, com visor de plástico e esquadro acoplado a uma das extremidades (Cameron, 1984), de acordo com os procedimentos sugeridos por Jelliffe (1966); as dobras cutâneas tricipital, bicipital, subescapular e suprailíaca foram verificadas por meio do equipamento Lange Skinfold Caliper, no lado direito do corpo, seguindo os métodos propostos por Jelliffe (1966) e Cameron (1984).

Quanto às características do hábito alimentar, foram questionados, durante a entrevista, o fracionamento da dieta, os tipos de refeições realizadas, as rejeições alimentares, os principais fatores determinantes do estilo de alimentação, além das alterações alimentares após o ingresso na universidade, nos finais de semana e em períodos de maior atividade acadêmica. Também investigou-se o consumo 
semanal dos grupos de alimentos constituintes da Pirâmide Alimentar (Bedinghaus \& Doughten, 1994). A análise foi realizada considerando-se as freqüências $\leq 1$, de 2-4 e de 5-7 vezes por semana, não sendo objetivo estabelecer critério que definisse consumo habitual dos grupos de alimentos.

Após a verificação das medidas foi calculado o Índice de Massa Corporal (IMC), 0 qual baseia-se na relação peso corporal $(\mathrm{kg}) /$ estatura $\left(\mathrm{m}^{2}\right)$. 0 estado nutricional foi classificado de acordo com a proposta da World... (1995).

A análise do percentual de gordura corporal (\% GC) foi realizada pelo somatório das quatro dobras cutâneas, conforme o sugerido por Durnin \& Womersley (1974), considerando-se como \% GC acima do esperado $>25 \%$ e $>20 \%$ para os sexos feminino e masculino, respectivamente (Sigulem et al., 1995).

Os dad os foram processados e analisados em banco de dados criado por meio do software Epi Info (Dean et al., 1990). Foi aplicado o teste do qui-quadrado para estudar a asso ciação entre sexo e adequação do estado nutricional (considerando-se eutróficos e distróficos) e entre sexo e adequação do percentual de gordura corporal dos adolescentes eutróficos. Fixou-se o nível de rejeição em $5 \%(p \leq 0,05)$, sendo os valores significantes assinalados com um asterisco.

A participação dos adolescentes recém-ingressos na universidade neste estudo foi espontânea após terem sido esclarecidos sobre 0 mesmo, sendo-Ihes também livre a opção de abandoná-lo quando desejassem. Aqueles que apresentaram algum problema de saúde ou nutricional foram encaminhados a atendimento individualizado no Programa de Atenção à Saúde do Adolescente (PROASA), da própria instituição.

\section{RESULTA DOS E DISCUSSÃ O}

A maioria dos adolescentes estudados $(92,4 \%)$ era procedente da Região Sudeste. Cerca de $90 \%$ deles não residiam com os pais e/ou familiares, ou seja, moravam em alojamento da universidade, república, casa ou apartamento mantidos pela família, pensão, hotel ou pensionato (Tabela 1).

Tabela 1. Condição socioeconômica, demográfica e de saúde referida por adolescentes universitários, 1998-1999.

\begin{tabular}{lcc}
\hline Variáveis & $n$ & $\%$ \\
\hline Procedência & 171 & 92,4 \\
Sudeste & 14 & 7,6 \\
Outras regiões & 168 & 89,8 \\
Residem sem os pais e/ou familiares & 7 & 3,8 \\
Exercem atividade remunerada & 80 & 43,2 \\
Praticam algum esporte & 13 & 7,0 \\
Consomem cigarros & 136 & 73,5 \\
Consomem bebidas alcóolicas & 75 & 46,5 \\
Tinham problemas de saúde antes do ingresso na Universidade & 61 & 33,0 \\
Manifestaram problemas de saúde após o ingresso na Universidade & 25 & 13,5 \\
Fazem uso de medicamentos & & 65,9 \\
História de doenças familiares & 122 & 51,9 \\
Hipertensão & 96 & 42,7 \\
Doença cardiovascular & 79 & 39,5 \\
Diabetes & 73 & 28,6 \\
Câncer & 53 & 28,1 \\
Obesidade & 53 & 21,6 \\
Alcoolismo & 52 & \\
Derrame cerebral & 40 & \\
\hline
\end{tabular}


Conforme se verificou também, $78 \%$ dos estudantes que não moravam com os pais estavam vivenciando pela primeira vez essa experiência. Este distanciamento da família talvez possa favorecer a ruptura de alguns hábitos e a adoção de novos estilos de vida (Barros, 1991).

Poucos realizavam atividade remunerada, e, destes, todos o faziam eventualmente e sem vínculo empregatício. 0 período de estudo, diurno para a maioria $(83,3 \%)$, pode ser um obstáculo para a realização de atividade remunerada, sobretudo fixa.

Observou-se que menos da metade dos adolescentes tinha o hábito de praticar esportes, dos quais $67,5 \%$ o faziam três vezes ou menos durante a semana e $50,0 \%$ não ultrapassavam quatro horas e meia semanais de realização do esporte. Estes dados mostram a necessidade de se incentivar a prática orientada de atividade física entre estes indivíduos, buscando-se evitar o sedentarismo. No entanto, constatou-se que a locomoção se dava essencialmente por meio de bicicleta e a pé, e somente $2,7 \%$ dos entrevistados locomoviam-se exclusivamente por meio de ônibus ou automóvel. Este resultado está de acordo com as características da cidade interiorana onde o estudo foi realizado, na qual mesmo as distâncias maiores muitas vezes dispensam veículos motorizados. A atividade física ligada à locomoção oferece contribuições ao gasto energético e auxilia na promoção da saúde, devendo, portanto, ser estimulada, assim como a prática esportiva.

O consumo de álcool era bastante difundido entre osuniversitários estudados, embora a maioria $(64 \%)$ o fizesse esporadicamente. 0 tabagismo era menos comum, mas cerca de $54 \%$ dos fumantes consumiam cigarros diariamente.

Questionou-se aos adolescentes a existência de alterações no estilo de vida - incluindo a prática de atividade física e o consumo de álcool e de cigarros - após o ingresso na universidade. Verificou-se que $39,2 \%$ deles relataram redução ou abandono da prática de atividade física enquanto $27,1 \%$ referiram início ou intensificação da mesma. Quanto ao consumo de álcool, 24,3\% disseram tê-lo iniciado ou aumentado e 9,9\% afirmaram tê-lo abandonado ou reduzido. 0 consumo de cigarros foi iniciado ou aumentado em 6,6\% dos entrevistados e reduzido ou abandonado em $2,8 \%$ deles, segundo seus próprios relatos. Conforme se pode constatar, as alterações negativas superaram as positivas em relação aos três aspectos investigados, chamando a atenção para a necessidade de melhor caracterizar a possível influência do ambiente universitário no estabelecimento do estilo de vida destes estudantes.

Estudando adolescentes de 12 a 18 anos matriculados em colégios públicos do município de São Paulo, Priore (1998) relatou que cerca de $50,0 \%$ tinha o hábito de consumir bebidas alcoólicas e $77,1 \%$ destes apresentavam ingestão com periodicidade esporádica. A maior faixa etária e o ambiente universitário podem ser fatores determinantes da maior freqüência de consumo de álcool encontrada entre os jovens do presente estudo em comparação com os pesquisados por Priore (1998).

Em relação ao consumo de cigarros, os resultados obtidos neste trabalho correspondem aos dados encontrados no estudo de Priore (1998), no qual muitos adolescentes não fumavam. Entretanto, não se deve deixar de enfocar os riscos do tabagismo nos trabalhos de prevenção com esse grupo populacional pois cerca de $90 \%$ dos fumantes ficam dependentes da nicotina até os 19 anos de idade, época em que o indivíduo ainda se encontra na fase de construção de sua personalidade, sendo, muitas vezes, o alvo principal da indústria tabagística (Oliveira, 1998).

No tocante aos problemas de saúde, os mais citados, tanto anteriores quanto posteriores ao ingresso na universidade, foram os respiratórios (rinite, sinusite e gripe) e os gastrintestinais (azia, gastrite e obstipação intestinal). Dos estudantes que utilizavam medicamentos, $36 \%$ iniciaram o uso após ingressarem na universidade.

Cerca de $15 \%$ dos adolescentes cujos relatos mencionaram problemas de saúde posteriores ao ingresso na universidade atribuíram 
tais distúrbios a causas emocionais, sugerindo que, talvez, mudanças ocorridas com o início da vida universitária possam ter comprometido o equilíbrio emocional e a saúde desse grupo de indivíduos.

Foi encontrada, em grande parte dos universitários estudados, história de doenças familiares positiva para doenças crônicas, reforçando a necessidade de se investir desde já na prevenção das mesmas, por meio da adesão a um estilo de vida mais saudável.

Analisando-se algumas características do hábito alimentar, pôde-se constatar que quase $60,0 \%$ dos adolescentes estudados não tinham o hábito de realizar as três refeições consideradas principais (desjejum, almoço e jantar). A realização do jantar tradicional, ou seja, da refeição noturna composta por alimentos presentes no típico almoço do brasileiro, era hábito apenas de 15,9\% dos entrevistados, sendo comumente substituído por lanches. Os resultados apontam para a necessidade de se trabalhar mais o consumo destes alimentos entre estes jovens, no sentido de transmitir-Ihes a importância de lanches balanceados e de ensinar-lhes receitas nutritivas. Tal conduta em relação aos lanches seria mais válida do que associá-los invariavelmente a um risco nutricional, recomendando sua eliminação da alimentação.

0 hábito de omitir o desjejum ocorria em cerca de $37,0 \%$ da população estudada. Analisando os resultados de um levantamento dietético com estudantes britânicos de 15 a 25 anos, Bull \& Phil. (1992) constataram que 18,0\% deles nunca faziam o desjejum. Huang et al. (1994) analisaram o padrão alimentar de estudantes universitários entre 19 e 20 anos e registraram omissão desta refeição por 22,0\% deles. Segundo Andersen et al. (1995), em pesquisa com adolescentes noruegueses com idade média de 18 anos, 13,4\% dos estudados realizavam o desjejum duas vezes ou menos por semana. Estes apresentaram um percentual maior de energia fornecida por gordura e açúcar e uma ingestão mais baixa de nutrientes em comparação com os que realizavam o desjejum mais freqüentemente.
Questionou-se sobre possíveis rejeições alimentares e cerca de $92,0 \%$ dos entrevistados mencionaram algum alimento. 0 grupo das hortaliças foi o mais referido, e $79,5 \%$ dos estudantes citaram pelo menos um alimento deste grupo. Tal resultado coincide com outros estudos realizados com adolescentes - nos quais componentes desse grupo lideram as listas de alimentos mais rejeitados (Huang et al., 1994; Priore, 1998) - e aponta para a necessidade de se trabalhar formas alternativas de aumentar a variabilidade na ingestão de vegetais (bolos, tortas, suflês, sucos, sopas, sanduíches, entre outros), buscando-se a harmonia entre o paladar dos jovens e estas importantes fontes de vitaminas, minerais e fibras. 0 grupo das frutas, que também fornece quantidades importantes desses nutrientes, foi 0 quarto mais citado, e $25,4 \%$ dos adolescentes disseram rejeitar uma ou mais frutas.

Analisando-se os dados acerca do consumo dos grupos de alimentos, verifica-se que aproximadamente $72 \%$ dos adolescentes mencionaram consumir hortaliças cinco ou mais vezes na semana. Entretanto, para uma melhor análise da ingestão dietética desses indivíduos e diante dos resultados referentes às rejeições alimentares, há a necessidade de se aplicar inquéritos sobre a freqüência de consumo dos alimentos. Esse inquérito permitirá averiguar a ocorrência ou não de monotonia alimentar, a qual pode estar relacionada a maior risco de carências nutricionais, caso haja pouca variabilidade no consumo de alimentos constituintes de cada grupo (Tabela 2).

Conforme se pode observar também (Tabela 2 ), a maior parte dos entrevistados $(75,1 \%$ ) relatou consumir frutas quatro vezes ou menos durante a semana. Em relação ao grupo das gorduras e doces, $46,5 \%$ disseram ingerir cinco vezes ou mais. $A$ análise da freqüência de consumo dos grupos de alimentos, neste caso, possibilitou verificar a ingestão insuficiente de frutas em grande parte dos adolescentes estudados. Ressalta-se também a ingestão freqüente de alimentos doces e gordurosos, pois 
Tabela 2. Consumo alimentar de adolescentes universitários, 1998-1999.

\begin{tabular}{|c|c|c|c|c|c|c|}
\hline \multirow{3}{*}{ Grupos } & \multicolumn{6}{|c|}{ Vezes por semana } \\
\hline & \multicolumn{2}{|c|}{$\leq 1$} & \multicolumn{2}{|c|}{$2-4$} & \multicolumn{2}{|c|}{$5-7$} \\
\hline & $n$ & $\%$ & $n$ & $\%$ & $n$ & $\%$ \\
\hline Cereais & - & - & - & - & 185 & 100,0 \\
\hline Hortaliças & 9 & 4,9 & 43 & 23,2 & 133 & 72,0 \\
\hline Frutas & 65 & 35,1 & 74 & 40,0 & 46 & 25,0 \\
\hline Laticínios & 8 & 4,3 & 27 & 14,6 & 150 & 81,1 \\
\hline Carnes/ovos/leguminosas & - & - & 5 & 2,7 & 180 & 97,3 \\
\hline Gorduras/doces & 9 & 4,9 & 90 & 48,6 & 86 & 46,5 \\
\hline
\end{tabular}

não foram considerados açúcar e óleo de adição no questionamento sobre o consumo do grupo das gorduras e doces. Esses resultados, em conjunto, contribuem para um perfil dietético que pode oferecer riscos à saúde atual e futura desta população.

Quando os adolescentes foram questionados sobre os principais fatores determinantes de seu estilo de alimentação, os três mais selecionados foram preferência pessoal $(48,1 \%)$, condição financeira $(42,2 \%$ ) e preocupação com a saúde $(37,8 \%)$. Constata-se, assim, que a preferência pessoal é o principal critério das escolhas alimentares para a maioria dos estudantes, reforçando a importância do processo de formação do hábito alimentar na definição das rejeições e freqüências de consumo verificadas. Como a adolescência, segundo a World Health... (1995), pode proporcionar oportunidades finais para a implementação de atividades ligadas à prevenção de doenças, é necessário e justificável investir na tentativa de melhoria do padrão alimentar dos indivíduos aqui estudados.
Quase 50\% dos entrevistados responderam estar ingerindo maior quantidade de alimentos após terem ingressado na universidade. Este resultado deve ser analisado juntamente com os dados acerca do consumo alimentar semanal, pois é mais importante saber quais alimentos passaram a ser consumidos ou tiveram sua ingestão aumentada do que simplesmente constatar o aumento do consumo alimentar após o ingresso na universidade (Tabela 3).

Aos adolescentes com alteração da ingestão nos finais de semana e períodos de maior atividade acadêmica, questionou-se quais alimentos passaram a ser consumidos ou tiveram o consumo aumentado nestes dias. De acordo com os resultados, a ingestão de "guloseimas" (doces, chocolates, biscoitos, refrigerantes e outros) é comum em ambos os períodos. Nos finais de semana, chama a atenção a grande parcela que lança mão da praticidade dos vários tipos de alimentos instantâneos, principalmente macarrão. Além da praticidade, também parece ser esta a opção de menor custo para substituir o jantar, o qual não é oferecido pelo restaurante universitário

Tabela 3. Alterações da ingestão alimentar de adolescentes universitários, 1998-1999.

\begin{tabular}{|c|c|c|c|c|}
\hline \multirow{2}{*}{ Circunstâncias / Alterações } & \multicolumn{2}{|c|}{ Aumento } & \multicolumn{2}{|c|}{ Redução } \\
\hline & n & $\%$ & $\mathrm{n}$ & $\%$ \\
\hline Após o ingresso na Universidade - alteração na quantidade total ingerida & 88 & 47,6 & 55 & 29,7 \\
\hline Em períodos de maior atividade acadêmica - alteração no fracionamento da dieta & 46 & 24,9 & 48 & 25,9 \\
\hline Em períodos de maior atividade acadêmica - alteração no volume das refeições & 41 & 22,2 & 35 & 18,9 \\
\hline Nos finais de semana - alteração no fracionamento da dieta & 82 & 44,3 & 38 & 20,5 \\
\hline Nos finais de semana - alteração no volume das refeições & 66 & 35,7 & 14 & 7,6 \\
\hline
\end{tabular}


nos finais de semana. Considerando-se estas facilidades operacionais e financeiras deve-se ficar atento para esta opção não se transformar em um hábito, pois a realização de refeições incompletas é uma prática tanto mais prejudicial quanto mais freqüente.

A ingestão acentuada de doces, chocolates e biscoitos em períodos de maior atividade acadêmica tanto pode ser reflexo da escassez de tempo para realizar refeições completas nessas circunstâncias, como pode sugerir indícios de compulsão alimentar em alguns adolescentes que, ansiosos, podem transformar a alimentação em "válvula de escape" para as situações de estresse físico e mental.

Foi verificado alto percentual de eutróficos, segundo o Índice de Massa Corporal. Entre os distróficos, a maioria $(69,2 \%)$ apresentava sobrepeso. Estas proporções de eutrofia superior à distrofia e de sobrepeso superior à baixo peso co incidem com as encontradas para a população adolescente na Pesquisa Nacional sobre Saúde e Nutrição (PNSN), realizada em 1989 (Neutzling, 1998), e com os dados de adolescentes de 12 a
18 anos, estudantes da rede pública estadual do município de São Paulo (Priore, 1998) (Tabela 4).

Entre os indivíduos com sobrepeso também estão incluídos aqueles que tiveram o diagnóstico de obesidade, segundo a classificação da World Health...(1995), sendo o percentual destes equivalente ao dobro do percentual daqueles (1,6\% e 3,2\%, respectivamente).

Apesar de não ter sido encontrada diferença estatisticamente significante, a prevalência de sobrepeso foi maior entre os jovens do sexo masculino, semelhantemente aos estudos de Pereira (1998) e Priore (1998), avaliando adolescentes do Rio de Janeiro e São Paulo, respectivamente.

Ainda que a avaliação pôndero-estatural tenha revelado um alto percentual de eutrofia, quase $60 \%$ dos eutróficos apresentaram \% GC acima do esperado para sua idade e sexo, situação prevalente sobretudo entre os do sexo feminino $(p<0,001)$. Tal resultado demonstra a importância da análise da composição corporal para um diagnóstico nutricional preciso deste grupo etário (Tabela 5).

Tabela 4. Estado nutricional de adolescentes universitários, de acordo com o sexo, 1998-1999.

\begin{tabular}{|c|c|c|c|c|c|c|}
\hline \multirow{3}{*}{ Estado nutricional } & \multicolumn{4}{|c|}{ Sexo } & & \\
\hline & \multicolumn{2}{|c|}{ Masculino } & \multicolumn{2}{|c|}{ Feminino } & \multicolumn{2}{|c|}{ Total } \\
\hline & $\mathrm{n}$ & $\%$ & $\mathrm{n}$ & $\%$ & $n$ & $\%$ \\
\hline Eutróficos & 72 & 91,2 & 100 & 94,3 & 172 & 93,0 \\
\hline Baixo peso & 2 & 2,5 & 2 & 1,9 & 4 & 2,2 \\
\hline Sobrepeso & 5 & 6,3 & 4 & 3,8 & 9 & 4,8 \\
\hline Total & 79 & 100,0 & 106 & 100,0 & 185 & 100,0 \\
\hline
\end{tabular}

Nota $=$ Teste do qui-quadrado $p=0,39$.

Tabela 5. Percentual de gordura corporal (\% GC) de adolescentes universitários eutróficos, de acordo com o sexo, 1998-1999.

\begin{tabular}{|c|c|c|c|c|c|c|}
\hline \multirow{3}{*}{$\% \mathrm{GC}$} & \multicolumn{4}{|c|}{ Sexo } & & \\
\hline & \multicolumn{2}{|c|}{ Masculino } & \multicolumn{2}{|c|}{ Feminino } & \multicolumn{2}{|c|}{ Total } \\
\hline & $n$ & $\%$ & $n$ & $\%$ & $n$ & $\%$ \\
\hline Adequado & 54 & 77,2 & 17 & 17,0 & 71 & 41,3 \\
\hline Elevado & 18 & 22,8 & 83 & $83,0 *$ & 101 & 58,7 \\
\hline Total & 72 & 100,0 & 100 & 100,0 & 172 & 100,0 \\
\hline
\end{tabular}

Nota $=(*)$ Diferença estatisticamente significante em relação ao sexo masculino; Teste do qui-quadrado $p=0,001$. 


\section{O N C LUSÃ O}

Apesar de quase $40 \%$ dos adolescentes estudados terem citado a preocupação com a saúde como um dos fatores determinantes do seu estilo de alimentação, os resultados apontam para um provável desequilíbrio da dieta de grande parcela desta população, considerando-se a omissão de refeições principais e a inobservância de uma ingestão adequada dos alimentos dos vários grupos. Simultaneamente aos erros alimentares, o estilo de vida adotado, principalmente após o ingresso na universidade, bem como a história de doenças familiares, contribuem como fatores de risco à saúde desses estudantes. A inadequação da dieta pode estar relacionada ao perfil antropométrico encontrado, refletindo-se, possivelmente, nos altos percentuais de gordura corporal observados.

Tendo-se constatado que peso normal não é sinônimo de composição corporal satisfatória entre estes adolescentes e que, mesmo alegando serem preocupados com a saúde, parecem não estar assumindo comportamentos efetivos neste aspecto, torna-se premente a necessidade de se direcionar ações individuais e coletivas de assistência a esta população específica.

\section{REFERÊ NCIAS B I B L I O G RÁ F I C A S}

ANDERSEN, L.F., NES, M., SANDTAD, B., BJORNEBOE, G., DREVON, C.A. Dietary intake among Norwegian adolescents. European Journal of Clinical Nutrition, London, v.49, p.555-564, 1995.

BARROS, R. $O$ adolescente e a família. In: MAAKAROUN, M.F., SOUZA, R.P., CRUZ, A.R. Tratado de adolescência: um estudo multidisciplinar. Rio de Janeiro : Cultura M édica, 1991. p.55-59.

BASTOS, A.C. Adolescência feminina. São Paulo : Atheneu, 1992. 105p.

BEDINGHAUS, J., DOUGHTEN, S. Childhood nutrition: from breastmilk to burgers. Primary Care Clinics in
Office Practice, Philadelphia PA, v.21, n.4, p.655-671, 1994.

BLUM, R.W.M. Risco e resiliência: sumário para desenvolvimento de um programa. Adolescência Latinoamericana, v.1, n.1, p.16-19, 1997.

BULL, N.L., PHIL, M. Dietary habits, food comsuption and nutrient intake during adolescence. Journal of Adolescent Health, New York, v.13, n.5, p.384-388, 1992.

CAMERON, N. The measurement of human growth. Austrália : Croom-Helm, 1984. 182p.

CHIPKEVITCH, E. A dimensão psicossocial. In: CHIPKEVITCH, E. Puberdade e adolescência: aspectos biológicos, clínicos e psicossociais. São Paulo : Roca, 1995. p.111-161.

DEAN, A.G., DEAN, J.A., BURTON, A.H., DICKER, R.C. Epi Info, version 5.01: a word processing, database, and statistics program for epidemiology on microcomputers [programa de computador]. Georgia : Center for Disease Control, 1990.

DURNIN, J.V.G., WOM ERSLEY, P. Body fat assessed from total body density and its estimation from skinfold tickness: measurement in 481 men and women aged from 16 to 72 years. British Journal of Nutrition, London, v.32, n.1, p.77-79, 1974.

FRISANCHO, A.R. Anthropometric standards for the assessment of growth and nutritional status. Ann Arbor MI : University of Michigan, 1989. 189p.

HUANG, Y., SONG, W.O., SCHEM M EL, R.A., HOERR, S. What do college students eat? Food selection and meat pattern. Nutrition Research, Elmsford, v.14, n.8, p.1143-1153, 1994.

JACOBSON, M.S. Nutrição na adolescência. Anais Nestlé, São Paulo, v.55, n.24, p.24-33, 1998.

JELLIFFE, D.B. The assessment of the nutritional status of the community. Geneva, 1966. 271p. (Série de Monografias, 53).

KNOBEL, M., SANTOS, M.G.P. 0 adolescente e o profissional de saúde. In: MAAKAROUN, M.F., SOUZA, R.P., CRUZ, A.R. Tratado de adolescência: um estudo multidisciplinar. Rio de Janeiro : Cultura M édica, 1991. p.145-155.

NEUTZLING, M.B. Sobrepeso em adolescentes brasileiros: Brasil, PNSN-1989. São Paulo, 1998. 
Dissertação (Mestrado) - Escola Paulista de M edicina, Universidade Federal de São Paulo, 1998.

OLIVEIRA, M.G.R. 0 tabagismo em crianças e adolescentes. Boletim Informativo SM P, n.3, dez., 1998.

ORGANIZACIÓN M UNDIALDELA SALUD. Necesidades de salud de los adolescentes. Ginebra, 1977. 55p. (Série de Informes Técnicos, 609).

PEREIRA, R.A. Avaliação antropométrica do estado nutricional. In: SICHIERI, R. Epidemiologia da obesidade. Rio de Janeiro : UERJ, 1998. p.43-64.

PRIORE, S.E. Composição corporal e hábitos alimentares de adolescentes: uma contribuição à interpretação dos indicadores de estado nutricional. São Paulo, 1998. 202p. (Doutorado) - Escola
Paulista de Medicina, Universidade Federal de São Paulo, 1998.

SIGULEM, D.M., VEIGA, G.V., PRIORE, S.E. Obesidade em adolescentes de baixa renda. In: FISBERG, M. Obesidade na infância e adolescência. São Paulo : Fundo Editorial BYK, 1995. p.80-83.

SOUZA, R.P. Abordagem na adolescência. In: SOUZA, R.P., MAAKAROUN, M .F. M anual de adolescência. São Paulo : Sociedade Brasileira de Pediatria, 1989. p.1-7.

WORLD HEALTH ORGANIZATION. Physical status: the use and interpretation of anthropometry. Geneva, 1995. 452p.

Recebido para publicação em 24 de outubro de 2000 e aceito em 26 de outubro de 2001. 